\title{
Practical Considerations towards Repeatable Measurements in Force Sensing Resistors (FSRs) ${ }^{\dagger}$
}

\author{
Leonel Paredes-Madrid ${ }^{1}$,* , Arnaldo Matute ${ }^{1}$, Elkin I. Gutiérrez Velásquez ${ }^{1}$ and \\ Carlos A. Parra Vargas ${ }^{2}$ \\ 1 Faculty of Electronic and Biomedical Engineering, Universidad Antonio Nariño, Tunja 150001, Colombia; \\ arnaldo.matute@uan.edu.co (A.M.); elkin.gutierrez@uan.edu.co (E.I.G.V.) \\ 2 Grupo de Física de Materiales (GFM), Universidad Pedagógica y Tecnológica de Colombia, Tunja 150003, \\ Colombia; carlos.parra@uptc.edu.co \\ * Correspondence: paredes.leonel@uan.edu.co \\ + Presented at the 5th International Symposium on Sensor Science (I3S 2017), Barcelona, Spain, \\ 27-29 September 2017.
}

Published: 14 December 2017

The usability of Force Sensing Resistors (FSRs) has been thoroughly demonstrated in applications demanding non-invasive force measurements, such as Gait Analysis (GA) and Object Manipulation (OM). The FSRs benefit from its light weight, customizable dimensions and low cost. Nonetheless, some authors have reported sensitivity degradation in FSRs when loaded to cyclic forces; this condition is a major concern that limits the extensive usage of FSRs. With the aim of replicating the conditions that cause such sensitivity degradation, a testbench has been built to exert dynamic force profiles over FSRs. The experimental tests were performed over FlexiForce A201-1 and Interlink FSR 402 sensors manufactured by Tekscan, Inc. and Interlink Electronics, Inc., respectively. It was found that sensitivity degradation occurs only when the sensor is cyclically loaded and the sourcing voltage is greater than $1.5 \mathrm{~V}$. Conversely, by setting a sourcing voltage below $1.5 \mathrm{~V}$ sensitivity degradation was not observed despite high-frequency mechanical forces. The underlying basis for this undesired phenomenon is not fully understood yet, but practical considerations are presented to avoid sensitivity degradation and to yield repeatable measurements; this is of great importance in certain applications with cyclic loading such as GA and OM.

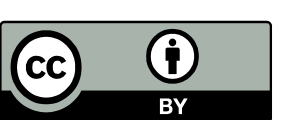

(C) 2017 by the authors. Licensee MDPI, Basel, Switzerland. This article is an open access article distributed under the terms and conditions of the Creative Commons Attribution (CC BY) license (http://creativecommons.org/licenses/by/4.0/). 